\title{
Gallensteine - Therapie
}

\author{
R. Jakobs, J. F. Riemann \\ Medizinische Klinik C, Klinikum der Stadt Ludwigshafen gGmbH
}

\section{Cholezystolithiasis Therapie}

Bei asymptomatischer Cholezystolithiasis besteht, von definierten Ausnahmen (Tab.1) abgesehen, prinzipiell keine Indikation zur Therapie (27).

Beim symptomatischen Gallensteinträger ist hingegen eine Therapieindikation gegeben, da in dieser Gruppe in 1-2\% der Fälle pro Jahr mit Komplikationen und in ca. 30\% mit Rezidivbeschwerden zu rechnen ist $(3,9,21,27)$.

Die laparoskopische Cholezystektomie ist die Standardtherapie der symptomatischen Cholezystolthiasis (25). Gegenüber der konventionellen Cholezystektomie hat die laparoskopische Technik einige Vorteile. Der Patient hat postoperativ weniger Schmerzen und kann früher mobilisiert werden, das kosmetische Ergebnis ist besser. Unter volkswirtschaftlichen Aspekten sind die kürzere Krankenhausaufenthaltsdauer und der raschere Wiedereintritt ins Erwerbsleben erwähnenswert. Die laparoskopische Cholezystektomie ist wahrscheinlich auch bezüglich der Komplikations- und Letalitätsdaten gegenüber der offenen Technik im Vorteil $(2,27,35)$.

Mehrere große Untersuchungen ergaben eine operationsassoziierte Letalität von etwa $0,08 \%$ bis $0,18 \%$ und postoperative Komplikationen (insbes. Blutung, Gallengangsverletzung mit/ohne Fistel, mit/ohne Stenose; Wundinfektion, Darmverletzung) bei etwa 3\% der Patienten. Die Konversionsrate zur konventionellen Cholezystektomie ist auf etwa 3-9\% der Eingriffe zu beziffern $(2,28,35)$.

Bei akuter Cholezystitis zeigen mehrere Untersuchungen den Vorteil der frühelektiven Operation innerhalb der ersten 72 Stunden $(2,27,28,33)$. In den meisten Fällen ist die dabei die laparoskopische Technik einsetzbar $(8,13)$. Bei gangränöser Cholezystitis, schweren Gerinnungsstörungen, unklaren anatomischen Strukturen und dem seltenen Mirizzi-Syndrom ist die Konversion zur offenen Cholezystektomie meist nicht vermeidbar $(8,36)$.

kurzgefasst: Therapie der Wahl der symptomatischen Cholezystolithiasis ist die Cholezystektomie. Auch bei akuter Cholezystitis kann die Cholezystektomie meist laparoskopisch erfolgen; sie sollte in dieser Situation frühelektiv (innerhalb der ersten 72 Stunden) durchgeführt werden.

Voraussetzungen für die orale Lysetherapie sind kalkfreie (Röntgen-negative) Steine mit einem Durchmesser bis $5 \mathrm{~mm}$ (in Einzelfällen bis $10 \mathrm{~mm}$ ) und eine gute Gallenblasenkontraktilität (> 60\% nach Reizmahlzeit). Mit einer oralen Therapie mit Ursodesoxycholsäure $(10-15 \mathrm{mg} / \mathrm{kg}$ ) wurden Steinfreiheitsraten bis zu $80 \%$ $(26,27)$ erzielt. Die Kombination mit Chenodesoxycholsäure verbessert nicht die Steinfreiheitsrate, erhöht allerdings die Rate an Nebenwirkungen (insbes. Diarrhoe, Transaminasenanstieg) (26).

Dtsch. Med. Wschr. 2001; 126: 883-885

(c) Georg Thieme Verlag Stuttgart · New York
Tab.1 Mögliche Indikationen zur Cholezystektomie bei operablen, asymptomatischen Patienten mit Cholezystolithiasis (nach 27).

- Porzellangallenblase

- gleichzeitiges Vorliegen von schnell wachsenden oder über $1 \mathrm{~cm}$ großen Gallenblasenpolypen

- Gallenblasensteine über $3 \mathrm{~cm}$ Durchmesser (insbes. Männer)

- bei speziellen abdominellen Eingriffen (z.B. lleumresektion, ausgedehnte Dünndarmresektionen, Transplantationen)

Tab. 2 Voraussetzungen für die konservativeTherapie von Gallenblasensteinen.

- typische biliäre Symptomatik

- keine Komplikationen

- gute Gallenblasenfunktion (mind. 60\% Kontraktion)

- durchgängiger Ductus cystikus

- nicht-verkalkte Steine

Die orale Lysetherapie sollte nach aktuellen Empfehlungen mindestens 3 Monate nach sonographisch verifizierter Steinfreiheit fortgesetzt werden (27). Die 5-Jahres-Rezidivrate der oralen Lysetherapie liegt allerdings bei bis zu $50 \%(25,27)$.

Die extrakorporale Stoßwellenlithotripsie (ESWL) ist ein etabliertes konservatives Verfahren zur Therapie der symptomatischen Cholezystolithiasis (31). Sind die in Tab.2 genannten Voraussetzungen erfüllt, konnten die höchsten Steinfreiheitsraten bei Solitärsteinen bis $20 \mathrm{~mm}$ Durchmesser oder bis 3 Steinen mit vergleichbarem Gesamtvolumen erzielt werden $(27,31)$. Zur schnelleren Beseitigung der verbliebenen Steinfragmente wird die ESWL üblicherweise mit einer UDC-Monotherapie wie bei der oralen Lysetherapie kombiniert.

Wird nach konservativem Therapieverfahren ein asymptomatischer Rezidivstein gefunden, ist nach derzeitiger Empfehlung keine erneute Therapieindikation gegeben. Beim symptomatischen Rezidivstein sollte hingegen eine laparoskopische Cholezystektomie erfolgen $(20,27)$.

Andere semiinvasive Verfahren wie die Cholezystostomie mit Steinentfernung bei Belassung der Gallenblase (20) und die direkte perkutane Lyse mittels Methyl-tert-butyl-Ether (MTBE) (14) haben sich in der klinischen Routine nicht durchgesetzt.

kurzgefasst: Konservative Behandlungsoptionen bei der symptomatischen Cholezystolithiasis sind die medikamentöse Lysetherapie und die extrakorporale Stoßwellenlithotripsie. Beide Verfahren weisen eine gute primäre Erfolgsrate, im Langzeitverlauf aber auch eine hohe Steinrezidivrate auf und sind auf unkomplizierte Fälle beschränkt. Die asymptomatische Cholezystolithiasis ist in der Regel keine Indikation zur Therapie. Ausnahmen bestehen z. B. bei Porzellangallenblase, gleichzeitig vorliegenden Gallenblasen- polypen $(>1 \mathrm{~cm})$ oder bei speziellen abdominellen Eingriffen, wie der Resektion des terminalen lleums. 


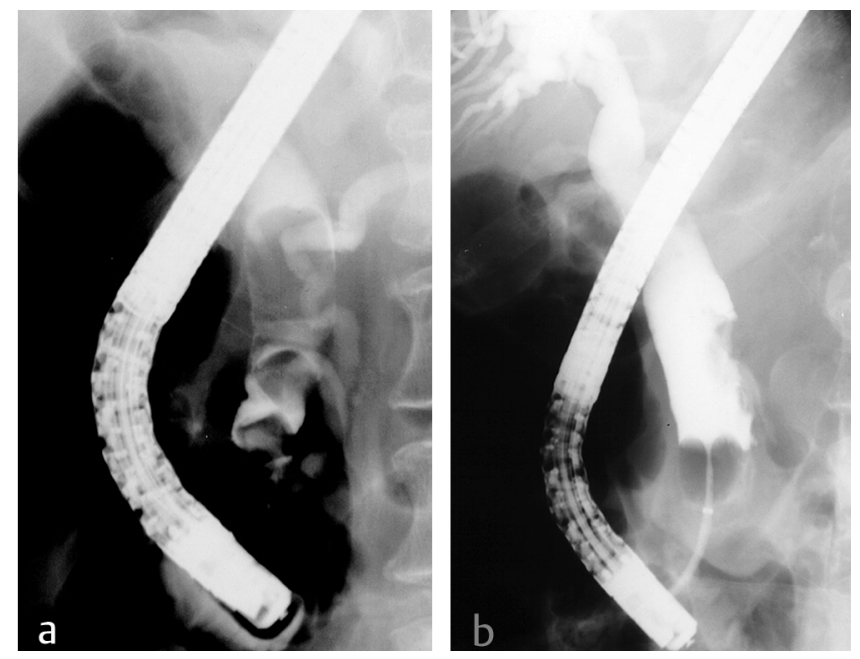

Abb.1 a: Nachweis mehrerer Choledochuskonkremente. b: Nach mechanischer Lithotripsie und Fragmentextraktion mit dem Dormiakorb weitgehend steinfreie Gallenwege.

\section{Cholangiolithiasis}

\section{Therapie}

Bei der Therapie der Cholangiolithiasis muss zwischen den Behandlungskonzepten bei Choledocholithiasis und Hepatolithiasis unterschieden werden. Die Standardtherapie der Choledocholithiasis ist die endoskopische Papillotomie $(6,22)$ und anschließende Steinextraktion mittels Korb oder Ballon. Durch diese Standardtechniken werden Steinfreiheitsraten zwischen $70 \%$ und 90\% erreicht (Abb.1). Berücksichtigt werden muss dabei das typische Komplikationsprofil der endoskopischen Papillotomie des Gallenganges mit den Risiken Pankreatitis (0,3-4\%), Blutung (2$4 \%$ ) oder seltener retroduodenale Perforation und Cholangitis. Die methodenbedingte Letalität erreicht - auch in Abhängigkeit von der Erfahrung des Untersuchers - 0,2 bis etwa 1\% $(12,23)$. Das endoskopische Vorgehen ist dabei bezüglich der Rate schwerer Komplikationen im Vorteil zu historischen Daten der operativen, offenen Gallengangsrevision und hat naturgemäß eine kürzere Hospitalisationsdauer zur Folge. Ein Steindurchmesser $>15 \mathrm{~mm}$, ein Missverhältnis zwischen Steindurchmesser und Gallengangsweite, eine ungünstige Steinlage (intrahepatisch, vor Stenosen) und eine ungünstige anatomische Situation, z.B. Duodenalstenose oder Billroth-II-Magenresektion sind typische Faktoren, die eine primäre Steinextraktion verhindern. Zur Therapie dieser definitionsgemäß komplizierten - Choledocholithiasis sind weitere Techniken erforderlich.

Verfahren der ersten Wahl in der Behandlung des komplizierten Gallengangssteines ist die mechanische Lithotripsie (27), die Anfang der 80er Jahre entwickelt wurde (29). Vorteile sind die niedrigen Kosten, die einfache Verfügbarkeit und ein hoher Effizienzgrad mit Erfolgsraten von etwa 60-80\%. Diese Methode setzt voraus, dass die komplizierten Gallengangssteine im Korb eingefangen werden können, was naturgemäß durch Steindurchmesser und Steinlage limitiert wird (32).

Aufwendigere Methoden der intra-, wie extrakorporalen Lithotripsie (28) werden überwiegend in Endoskopiezentren vorgehalten, weil dort sowohl eine ausreichende Erfahrung des Therapeuten wie eine wirtschaftliche Nutzung der Geräte gewährleistet ist.

Die elektrohydraulische Lithotripsie (EHL) basiert auf dem Prinzip der Unterwasserfunkenentladung und erfordert wegen der potenziellen Gefahr der Gallengangsperforation bei Fehlapplikation den obligaten
Tab.3 Publizierte Daten zur elektrohydraulischen (EHL), zur laserinduzierten (LISL) und extrakorporalen Stoßwellen-Lithotripsie (ESWL) von Gallengangssteinen

\begin{tabular}{llll}
\hline Autor, Publikationsdatum & Technik & Patienten (n) & Steinfreiheit \\
\hline Hochberger, 1997 (15) & LISL & 60 & $87 \%$ \\
Jakobs, 1998(18) & LISL & 89 & $87 \%$ \\
Adamek, 1996(1) & EHL & 49 & $74 \%$ \\
Binmoeller, 1993(5) & EHL & 65 & $98 \%$ \\
Sackmann, 1991(31) & ESWL & 711 & $86 \%$ \\
Ellis, 2000(10) & ESWL & 83 & $83 \%$
\end{tabular}

Einsatz eines Cholangioskops zur direkten optischen Kontrolle. Wenn die Steine mit dem Cholangioskop erreicht werden können, gelingt in etwa $90 \%$ auch die Desintegration der Steine $(1,5,30)$.

Die laserinduzierte Stoßwellenlithotripsie (LISL) ist der EHL vergleichbar effektiv $(15,17)$. Durchgesetzt hat sich im europäischen Raum ein "intelligenter" Rhodamin-Laser. Dieser spezielle Laserlithotriptor hat ein integriertes Stein-Gewebe-Erkennungssystem, das den Einsatz ohne Cholangioskop ermöglicht, da es eine Abschaltung des Laserimpulses bei Fehlapplikation auf Gewebe gewährleistet $(15,17)$.

Neben diesen intrakorporalen, rein endoskopischen Verfahren steht die extrakorporale Stoßwellenlithotrispie (ESWL) zur Verfügung, die ähnliche Steinfreiheitsraten erzielt, wie die oben beschriebenen intrakorporalen Methoden $(1,5,30)$.

Alle angeführten Lithotripsietechniken haben eine ausgesprochen niedrige Komplikationsrate (27). Mit der Kombination der oben beschriebenen Techniken sind Steinfreiheitsraten von etwa 98\% bei der Behandlung der Choledocholithiasis zu erzielen (eigene Daten der Med. Klinik C / Ludwigshafen an über 2500 behandelten Patienten). Die Langzeitergebnisse nach endoskopischer Sphinkterotomie wegen Choledocholithiasis sind gut. Nach kompletter Steinextraktion ist nur in etwa $11,3 \%$ im Langzeitverlauf mit einem Steinrezidiv zu rechnen (34).

Für die wenigen Fälle, die endoskopisch nicht zu sanieren sind, stehen Alternativen zur Auswahl, die in Abstimmung mit dem kooperierenden chirurgischen Partner eingesetzt werden sollten.

Bei guter Operabilität und gleichzeitig bestehender Cholezystolithiasis ist die Cholezystektomie mit intraoperativer Therapie der Choledochussteine hilfreich. Ist der Patient bereits cholezystektomiert, kann bei schlechter transpapillärer Erreichbarkeit der Steine die perkutan-transhepatische Choledochusdrainage mit perkutaner Cholangioskopie zur Sanierung der Gallenwege eingesetzt werden.

Bei Cholangitis und primär nicht sanierbarer Choledocholithiasis ist eine temporäre endoskopische biliäre Endoprotheseneinlage sehr hilfreich (4). Bei lokal oder systemisch inoperablen Patienten und Versagen der endoskopischen Fragmentationstechniken stellt die biliäre Endoprothetik eine effektive Palliation dar. Die permanente Prothesenversorgung sollte aber wegen des Risikos der Sepsis bei Prothesenokklusion auf Einzelfälle beschränkt werden $(4,25,27)$.

Wird vor geplanter Cholezystektomie eine Choledocholithiasis diagnostiziert, wird ein sog. therapeutisches Splitting empfohlen. Die primäre endoskopische Extraktion der Gallengangssteine und die anschließende laparoskopische Cholezystektomie ist dabei nach den entsprechenden Leitlinien Vorgehen der Wahl (27). 
Die chirurgische Sanierung der extrahepatischen Gallenwege bei Choledocholithiasis ist nur in Ausnahmefällen erforderlich. Eine mögliche Option stellt die laparoskopische Cholezystektomie mit laparoskopischer Gallengangsrevision dar. Die ersten Erfahrungsberichte zeigen Erfolgsraten von etwa 70-95\% (7,27). Die Anwendung dieser relativ neuen Methode ist aber derzeit noch auf eine geringe Zahl chirurgischer Zentren reduziert. Die offene Choledochusrevision mit Choledochostomie scheint bei jüngeren Patienten im Stadium ASA-1 und -2 genauso effizient und sicher zu sein, wie die präoperative Papillotomie und Steinextraktion $(11,26)$. Bei akuter Cholangitis und mit steigendem Alter des Patienten erhöhen sich die Komplikationsrate und die operationsbedingte Letalität infolge offener Choledochusrevision deutlich und erreichen bis zu 10\% (26). Bei kleinen Gallengangsteinen und erhöhtem Risikoprofil des Patienten sollte - die Verfügbarkeit ausgewiesener Partner vorausgesetzt - individuell über eine postoperative, endoskopische Sanierung der Gallenwege entschieden werden.

\section{kurzgefasst: Die Therapie von Gallengangssteinen ist die Domä- ne der Endoskopie. Nach endoskopischer Papillotomie gelingt die Steinextraktion mittels Ballonkatheter oder Korb in mehr als $70 \%$ der Fälle. Für ungünstige anatomische Situationen, sehr große Steine oder bei einem Missverhältnis zwischen Stein- und Gangdurchmesser stehen mehrere intra- wie extrakorporale Verfahren zur Steinfragmentation zur Verfügung. Bei entspre- chenden apparativen wie personellen Voraussetzungen gelingt die Behandlung der Choledocholithiasis in mehr als $97 \%$ der Fälle auf endoskopischem Wege. Liegen gleichzeitig Gallenblasen- steine und eine Choledocholithiasis vor, wird derzeit das "thera- peutische Splitting" favorisiert, das eine endoskopische Behand- lung der Choledocholithiasis mit anschließender laparoskopi- scher Cholezystektomie beinhaltet. Mit neuen laparoskopischen Techniken ist die Kombination von laparoskopischer CHE und Choledochusrevision in gleicher Sitzung möglich.}

Das therapeutische Vorgehen bei der in einigen Regionen Asiens endemischen primären Hepatolithiasis soll wegen der Seltenheit in unseren Breiten nur kurz erörtert werden. In vielen dieser Fälle ist ein operativ-chirurgisches Verfahren erforderlich. Insbesondere bei gleichzeitiger Cholezystolithiasis, wenn die Steine auf ein Lebersegment begrenzt sind, zur lokalen Abszessbildung oder Segmentatrophie geführt haben oder der Verdacht auf ein cholangiozelluläres Karzinom besteht, bietet ein leberresezierender Eingriff eine potenziell kurative Option $(19,37)$.

Nach Voroperationen oder bei komplexen intrahepatischen Steinlagen, z.B. mit diffusem Befall mehrer Segmente ist heute der perkutan-transhepatische Zugang oder bei gleichzeitig erfolgender Cholezystektomie der perkutane Zugang über den TTrakt das Verfahren mit der höchsten Erfolgsrate. In verschiedenen Untersuchungen wurden eine Steinfreiheit bei bis zu $90 \%$ der behandelten Patienten erzielt, die Komplikationsrate ist bei etwa $5-8 \%$ anzusiedeln und betrifft vorwiegend Hämobilie und Cholangitis (53). Transpapilläre endoskopische Verfahren haben wegen der oft schlechten Erreichbarkeit der Steine infolge peripherer Lage, begleitender Stenosen oder vorhergehender operativer Eingriffe eine untergeordnete Bedeutung in der Behandlung der komplexen Hepatolithiasis (19).

kurzgefasst: Bei intrahepatischen Gallengangssteinen ist eine Kombination aus Leber-(Segment-) resektion (ggf. im Rahmen einer Cholezystektomie) und perkutan-endoskopischen Techniken die therapeutische Strategie mit der höchsten Erfolgsrate. Transpapilläre Techniken haben wegen der oft schlechten Erreichbarkeit der Steine bei der Hepatolithiasis eine untergeordnete Bedeutung.

\section{Literatur}

1 Adamek HE, Maier M, Jakobs R et al. Management of retained bile duct stones. Gastrointest Endosc 1996; 44: 40-47

2 Ärztekammer Nordrhein. Qualitätssicherung Chirurgie Nordrhein 1998 Cholelithiasis: 17.685 Behandlungsdokumentationen aus 154 Abteilungen,

3 Barbara LC, Sama AMM, Labate F et al. A population study on the prevalence of gallstone disease: the Sirmione study. Hepatology 1987; 7: 913-919

4 Bergman JJ, Rauws EA, Tijssen JG, Tytgat GN, Huibregtse K. Biliary endoprostheses in elderly patients with endoscopically irretrievable common bile theses in elderly patients with endoscopically irretrievable common bile

Binmoeller KF, Bruckner M, Thonke F, Soehendra N. Treatment of difficult Binmoeller KF, Bruckner M, Thonke F, Soehendra N. Treatment of difficult
bile duct stones using mechanical, electrohydraulic and extracorporeal shock wave lithotripsy. Endoscopy 1993; 25: 201-206

6 Classen M, Demling L. Endoskopische Sphinkterotomie der Papilla Vateri und Steinextraktion aus dem Ductus choledochus. Dtsch Med Wochenschr 1974; 99: 469-467

7 Crawford DL, Phillips EH. Laparoscopic common bile duct exploration. World J Surg 1999; 23: 343-349

8 Cuschieri A. Approach to the treatment of acute cholecystitis: Open surgical, laparoscopic or endoscopic? Endoscopy 1993; 25: 397-398

9 Egbert AM. Gallstone symptoms. Postgrad Med 1991; 90: 119-126

10 Ellis RD, Jenkins AP, Thompson RP, Ede RJ. Clearance of refractory bile duct stones with extracorporeal shock wave lithotripsy. Gut 2000; 47: 728-731

11 Förster R, Lindlar R et al. Ist die einseitige Cholezystektomie mit Choledochusrevision obsolet? Dtsch med Wschr 1990; 115: 563-569

12 Freeman ML, Nelson DB, Sherman S et al. Complications of endoscopic biliary sphincterotomy. N Engl J Med 1996; 335: 909-918

13 Garber SM, Korman J, Cosgrove JM, Cohen JR. Early laparoscopic cholecystectomy for acute cholecystitis. Surg Endosc 1997; 11: 347-350

14 Hellstern A, Leuschner U et al. Dissolution of gallbladder stones with methyl-tert-butyl ether and stone recurrence. Dig Dis Sci 1998; 43: 911-915

15 Hochberger J, Bayer J, May A et al. Laser lithotripsy of difficult bile duct stones: Results in 60 patients using a rhodamine $6 \mathrm{G}$ dye laser with optical stone tissue detection system. Gut 1998; 43: 823-829

16 Hultman CS, Herbst CA, McCall JM, Mauro MA. The efficacy of percutaneous cholecystostomy in critically ill patients. Am Surg 1996; 62: 263-269

17 Jakobs R, Adamek HE, Maier M et al. Fluoroscopically-guided laser lithotripsy versus extracorporeal shock wave lithotripsy for retained bile duct psy versus extracorporeal shock wave lithotripsy for retained

18 Jakobs R, Benz C, Martin WR, Arnold JC, Riemann JF. Laser lithotripsy of difficult bile duct stones. Gastroenterology 1998; 114: A4112

19 Jakobs R, Riemann JF. Hepatikolithiasis - endoskopisch-interventionelle Therapiemöglichkeiten. Chir Gastroenterol 1999; 15: 274-278

20 Janssen J, Johanns W, Weickert U et al. Long-term results after successful extracorporeal lithotripsy. Scand J Gastroenterol 2001; 36: 314-317

21 Jorgensen T. Prevalence of gallstones in a Danish population. Amer J Epidemiol 1987; 126: 912-921

22 Kawai K, Akasak Y, Murakami K et al. Endoscopic sphincterotomy the ampulla of Vater. Gastrointest Endosc 1974; 20: 148

23 Loperfido S, Angelini G, Benedetti G et al. Major early complications from diagnostic and therapeutic ERCP. Gastrointest Endosc 1998; 48: 1-10

24 Maxton DG, Tweedle DE, Martin DF. Retained common bile duct stones after endoscopic sphincterotomy. Gut 1995; 36: 446-449

25 May GR, Sutherland LR, Shaffer EA. Efficacy of bile acid therapy for gallstone dissolution. Aliment Pharmacol Ther 1993; 7: 139-148

26 Neoptolemos JP, Carr-Locke DL, Fossard DL. Prospective randomised study of preoperative endoscopy versus surgery alone for common bile duct stones. Brit med J 1987; 294: 470-474

27 Neubrand M, Sackmann M, Caspary WF et al. Leitlinien der Deutschen Gesellschaft für Verdauungs- und Stoffwechselkrankheiten zur Behandlung von Gallensteinen. Z Gastroenterol 2000; 38: 449-468

28 Richardson MC, Bell G, Fullarton GM. Incidence and nature of bile duct injuries following laparoscopic cholecystectomy. West of Scotland Laparoscopic cholecystectomy audit group. Br J Surg 1996; 83: 1356-1360

29 Riemann JF, Kohler B, Weber J, Schlauch D. Intraductal shock-wave lithotripsy in complicated common bile duct stones. Clin Invest 1992; 70: 148-151

30 Riemann JF, Seuberth K, Demling L. Clinical application of a new mechanical lithotripter for smashing common bile duct stones. Endoscopy 1982; 14: 226-230

31 Sackmann M, Pauletzki J, Sauerbruch T et al. The Munich gallbladder lithotripsy study. Ann Intern Med 1991; 114: 290-296

32 Schneider MU, Matek W, Bauer R et al. Mechanical lithotripsy of bile duct stones in 209 patients: effect of technical advances. Endoscopy 1988; 28: 248

33 Schwesinger WH, Sirinek KR, Strodel WE. Laparoscopic cholecystectomy for biliary tract emergencies. World J Surg 1999; 23: 334-342

34 Seifert E. Long-term follow-up after endoscopic sphincterotomy (EST). Endoscopy 1988; 20: 232-235

35 Steiner CA, Bass EB, Talamini MA, Pitt HA, Steinberg EP. Surgical rates and operative mortality for open and laparoscopic cholecystectomy in Maryland. N Engl J Med 1994; 330: 403-408

36 Targarona EM, Andrade E, Balague C, Ardid J, Trias M. Mirizzi's syndrome. Surg Endosc 1997; 11: 842-845

37 Yeh YH, Huang MH, Yang JC et al. Percutaneous transhepatic cholangioscopy and lithotripsy in the treatment of intrahepatic stones. A study with a 5year follow-up. Gastrointest Endosc 1995; 42: 13-18

\section{Korrespondenz}

Dr. Ralf Jakobs

Med. Klinik C

Klinikum der Stadt Ludwigshafen gGmbH

Bremserstraße 79

67063 Ludwigshafen 